\title{
Comparación de variables morfométricas y bioquímico-nutricionales de búfalas y bubillas de Corrientes, Argentina
}

\author{
Koza, G.A.; Mussart, N.B.; Hernando, J.; Konrad, J.L.; Crudeli, G.A. \\ Cátedras de Fisiología y Teriogenología, Facultad de Ciencias Veterinarias, Universidad Nacional del Nordeste, \\ Cabral 2139, Corrientes (3400), Argentina. E-mail: gakoza@vet.unne.edu.ar
}

\begin{abstract}
Resumen
Koza, G.A.; Mussart, N.B.; Hernando, J.; Konrad, J.L.; Crudeli, G.A.: Comparación de variables morfométricas y bioquímico-nutricionales de búfalas y bubillas de Corrientes, Argentina. Rev. vet. 28: 2, 108-115, 2017. El objetivo del trabajo fue analizar las diferencias de algunos parámetros hematológicos, bioquímicos y morfométricos entre bubillas y búfalas de dos establecimientos (Itatí y Empedrado) de la Provincia de Corrientes, Argentina. Se emplearon 60 hembras bubalinas, raza mediterránea, 15 por categoría. Se registró el peso vivo $(\mathrm{PV})$ y el perímetro torácico $(\mathrm{PT})$. Se tomaron muestras sanguíneas para valorar hematocrito (HTO), hemoglobina (HB), recuento de eritrocitos (RGR) y leucocitos (RGB), volumen corpuscular medio (VCM), hemoglobina corpuscular media (HCM) y concentración de HCM (CHCM). En el suero se estimaron urea, proteínas totales (PRT), albúminas (ALB), globulinas (GLOB), relación albúmina-globulina (RAG), colesterol (CT, C-HDL y C-.LDL), triglicéridos (TR), calcio (CA), fósforo (FOS), magnesio (MG), glucosa y actividad de lactato deshidrogenasa (LDH). Se utilizó un diseño experimental de arreglo factorial, donde la variable independiente fue la localidad y el tratamiento la categoría, valorándose la interacción de localidad $\mathrm{x}$ edad. Con el programa InfoStat se efectuó la estadística descriptiva y el ANOVA. La interacción significativa de algunas variables motivó el tratamiento por sus efectos simples. Las búfalas de Empedrado presentaron mayores PV, PT, neutrófilos, eosinófilos, CT, TR y urea, mientras que las bubillas tuvieron elevados linfocitos, CA, glucosa y LDH. Las búfalas de Itatí revelaron mayores PV, PT, HTO, RGR, CT y las hembras jóvenes elevados niveles de LDH. El efecto edad influyó, por lo cual las búfalas ostentaron mayores porcentajes de monocitos, PRT y GLOB, en tanto que en las bubillas fueron más altos RGB, FOS, C-HDL y C-LDL. Para el factor localidad los animales de Empedrado revelaron mayores niveles de HB, HCM, CHCM, RGR, monocitos y MG; los de Itatí mostraran elevadas PRT, ALB y RAG. No hubo diferencias para VCM y basófilos. La obtención de intervalos de referencia bioquímicos regionales asume importancia como herramienta para evaluar el estado nutricional y optimizar el diagnóstico de enfermedades.
\end{abstract}

Palabras clave: búfalos, crecimiento, nutrición, valores morfométricos y bioquímicos, producción animal.

\begin{abstract}
Koza, G.A.; Mussart, N.B.; Hernando, J.; Konrad, J.L.; Crudeli, G.A.: Comparison of differences between morphometrical and biochemical-nutritional parameters of buffaloes (adults and young females) from Corrientes, Argentina. Rev. vet. 28: 2, 108-115, 2017. The objective of this study was to analyze the differences in hematological, biochemical and morphometrical parameters between young and adult female buffaloes from two farms (Itatí and Empedrado) in Corrientes Province, Argentina. Sixteen Mediterranean buffaloes, 15 per category, were used. Weight and thoracic perimeter were recorded considering both geographic regions. Hematocrit, hemoglobin, erythrocyte and leukocyte counts, mean corpuscular volume, mean corpuscular hemoglobin, mean corpuscular hemoglobin concentration and relative leukocyte formula, were registered. Urea, total proteins, albumin, globulins, albumin-globulin ratio, cholesterol, triglycerides, calcium, phosphorous, magnesium, glucose and lactate dehydrogenase, were determined in serum. An experimental design of factorial arrangement was used, where the independent variable was the geographic region and the treatment the category, considering the interaction region $\mathrm{x}$ age. Descriptive statistics and analysis of variance were performed using InfoStat software. Empedrado buffaloes had higher values of live weight, total protein, neutrophils, eosinophils, cholesterol, triglycerides and urea, whereas young females had high values of lymphocytes, calcium, glucose and
\end{abstract}


lactate dehydrogenase. Buffaloes from Itatí revealed higher values of weight, hematocrit, erythrocytes and cholesterol. Young females showed high lactate dehydrogenase levels. Age of animals influenced values, as adult buffaloes had higher percentages of monocytes, total proteins and globulins, while young females had higher values of leucocytes and phosphorus. Considering the geographic region, animals from Empedrado revealed higher levels of hemoglobin, erythrocytes, monocytes and magnesium. Animals from Itatí showed high protein, albumin and albumin-globulin ratio. The mean corpuscular volume and basophils were not modified. Regional biochemical reference intervals are of great importance as a tool to evaluate blood nutritional indicators and to optimize the diagnosis of diseases.

Key words: buffalos, growth, nutrition, morphometrical and biochemical values, animal production.

\section{INTRODUCCIÓN}

De acuerdo a los datos aportados por el Servicio Nacional de Sanidad y Calidad Agroalimentaria (SENASA), en el año 2014 la población de búfalos en Argentina fue de 87.711 cabezas. Según categorías, los porcentajes variaron de la siguinete manera: búfalas $(42,4 \%)$, bubillas $(17,8 \%)$, bucerras $(11,7 \%)$, bucerros $(11,1 \%)$, bubillitos $(7,3 \%)$, bubillos $(5,3 \%)$, búfalos $(3,3 \%)$, butoritos $(0,7 \%)$ y buey búfalo $(0,4 \%)^{14}$.

La explotación bubalina se instaló en las zonas bajas del nordeste argentino, que actualmente concentra el $80 \%$ de la producción, sitios donde los vacunos no se adaptaban ${ }^{5}$. Las provincias con mayores poblaciones bubalinas son Formosa y Corrientes. Por su gran rusticidad, la especie bubalina aprovecha mejor el forraje de baja calidad, aún bajo condiciones climáticas adversas, manifestando una notable resistencia a las enfermedades; los búfalos ganan más peso con relación a los bovinos, por su mayor capacidad de digestión de celulosa, pastos fibrosos y groseros ${ }^{24}$.

El desarrollo reproductivo de las hembras jóvenes y el logro de una pubertad temprana, puede alcanzarse principalmente con una buena nutrición desde el momento del destete. La mejora en la eficiencia reproductiva y productiva del ganado bubalino, depende principalmente de la modificación de ciertos parámetros, que involucran principalmente la alimentación, el medio ambiente y el manejo ${ }^{8}$.

La composición bioquímica del suero sanguíneo refleja la situación metabólica de los tejidos animales, trastornos en el funcionamiento de los órganos, adaptación del organismo animal frente a alteraciones nutricionales y fisiológicas, como así también desequilibrios metabólicos específicos o de origen nutricional. Factores como la especie, raza, edad, sexo, hábitat, sistema de crianza y alimentación -entre otros- influyen sobre los resultados de la bioquímica sérica ${ }^{22}$.

Para la especie bubalina, los datos disponibles en la región son escasos, la mayoría generados por nuestro grupo de trabajo, debiendo recurrise a la bibliografia de otras latitudes como referencia. Por lo tanto, existe una creciente necesidad de obtener hallazgos clínicos y de laboratorio específicos para los búfalos, respetando las peculiaridades fisiológicas que le son inherentes a estos animales ${ }^{7}$, considerando las zonas geográficas donde se ubican las explotaciones.

El objetivo del trabajo fue analizar las diferencias de algunos parámetros hematológicos y bioquímiconutricionales, peso corporal y perímetro torácico entre bubillas de primer servicio y búfalas adultas, de las localidades de Itatí y Empedrado, en la Provincia de Corrientes, Argentina.

\section{MATERIAL Y MÉTODOS}

La toma de muestras se realizó a 60 búfalas raza Mediterránea, de dos establecimientos ganaderos, uno próximo a la localidad de Itatí y otro en cercanías del municipio de Empedrado. En cada lugar, se seleccionaron 15 animales de la categoría bubillas (hembras de aproximadamente 2,5 años de edad, próximas a recibir su primer servicio) y otros 15 de la categoría búfalas (hembras mayores de 4 años, vacías). Las condiciones de alimentación (campo natural), sanidad y manejo fueron similares en los todos casos.

Se procedió al registro del peso vivo (PV, en báscula) y el perímetro torácico (PT, medición con cinta métrica), así como a la toma muestras sanguíneas (por venopunción yugular). Con la sangre entera anticoagulada con EDTA se efectuó la valoración de hematocrito (HTO), hemoglobina (HB), recuento de glóbulos rojos (RGR) y blancos (RGB), valoración de índices hematimétricos (VCM: volumen corpuscular medio, HCM: hemoglobina corpuscular media, CHCM: concentración de hemoglobina corpuscular media) y fórmula leucocitaria relativa, de acuerdo a técnicas de laboratorio convencionales.

Con el suero obtenido se llevaron a cabo las valoraciones bioquímicas de algunos analitos incluidos en los perfiles nitrogenado (urea, proteínas totales-PRT, albúminas-ALB, globulinas-GLOB, relación albúminaglobulina-RAG), lipídico (colesterol total-CT, triglicéridos-TR, mineral (calcio-CA, fósforo inorgánico-FOS, magnesio-MG), glucosa y la enzima lactato deshidrogenasa (LDH), cuyas determinaciones se realizaron en el Laboratorio de Análisis Clínicos del Hospital de la Facultad de Ciencias Veterinarias de la UNNE, según 
técnicas convencionales, empleado reactivos Wiener Lab.

Para las estadísticas se utilizó un diseño experimental de arreglo factorial, donde la variable independiente fue la localidad (Itatí-Empedrado) y el tratamiento fue la edad (búfala-bubilla), valorándose la interacción localidad $\mathrm{x}$ edad. Las variables dependientes fueron: peso vivo, perímetro torácico, condición corporal, indicadores hematológicos y bioquímico-nutricionales. Con la ayuda del programa estadístico InfoStat Profesional (2016), se realizaron las estadísticas descriptivas, el análisis de la varianza y la diferencia entre medias (test de Tukey).

\section{RESULTADOS Y DISCUSIÓN}

Los resultados obtenidos para las variables analizadas se consignan en la Tabla 1. Como muchos de los parámetros explorados revelaron interacción significativa entre los efectos de la edad (categoría) y de la localidad (lugar de residencia), los mismos se trataron por sus efectos simples, es decir, edad por localidad (Tabla 2) y localidad por edad (Tabla 3).

El PV mostró una significativa interacción de edad por localidad $(\mathrm{p}=0,0003)$. Las búfalas adultas revelaron significativamente mayores valores de PV $(p=0,0001)$ que las bubillas de ambas localidades (Tabla 2). Considerando el efecto de la localidad, en la Tabla 3 puede observarse que las búfalas de Empedrado fueron más pesadas que las de Itatí $(p=0,003)$, mientras que estas últimas bubillas, ostentaron $\mathrm{PV}$ más elevados que las de Empedrado ( $\mathrm{p}=0,006$ ).

Generalmente, los búfalos alcanzan la pubertad cuando adquieren cerca el $55-60 \%$ de su peso corporal adulto (250 a $400 \mathrm{~kg}$ ), dependiendo del genotipo animal ${ }^{26}$. La capacidad que tienen los búfalos para lograr pesos elevados a edades tempranas, puede relacionarse con algunas características reproductivas, como la edad al primer servicio y la edad al primer parto; en un trabajo se recomienda que la actividad reproductiva de

Tabla 1. Efectos de la localidad y la edad, sobre variables morfométricas, hemáticas y bioquímicas.

\begin{tabular}{|c|c|c|c|c|c|c|c|}
\hline \multirow{2}{*}{ variable } & \multicolumn{2}{|c|}{ Itatí } & \multicolumn{2}{|c|}{ Empedrado } & \multirow{2}{*}{$\frac{\text { edad }}{\mathrm{p}}$} & \multirow{2}{*}{$\begin{array}{c}\text { local. } \\
\mathrm{p}\end{array}$} & \multirow{2}{*}{$\begin{array}{c}\text { interac. } \\
\mathrm{p}\end{array}$} \\
\hline & búfalas & bubillas & búfalas & bubillas & & & \\
\hline PV (kg) & $553,10 \pm 7,65 a$ & $421,20 \pm 10,82 b$ & $598,33 \pm 8,84 c$ & $397,00 \pm 884 b$ & $0,0001^{*}$ & 0,25 & $0,0003 *$ \\
\hline PT (cm) & $215,05 \pm 3,08 \mathrm{a}$ & $188,30 \pm 4,36 \mathrm{~b}$ & $216,73 \pm 3,56 a$ & $174,07 \pm 3,56 \mathrm{c}$ & 0,09 & $0,0001 *$ & $0,03^{*}$ \\
\hline HTO (\%) & $40,60 \pm 0,49 a$ & $37,40 \pm 0,70 \mathrm{~b}$ & $36,60 \pm 0,57 b$ & $36,87 \pm 0,57 b$ & $0,015^{*}$ & $0,0003^{*}$ & $0,004 *$ \\
\hline RGR (T/1) & $9,12 \pm 0,13 \mathrm{a}$ & $8,38 \pm 0,19 b$ & $8,93 \pm 0,15 b$ & $8,19 \pm 0,15 b$ & $0,003^{*}$ & $0,0004^{*}$ & $0,01^{*}$ \\
\hline $\operatorname{VCM}\left(\mathrm{u}^{3}\right)$ & $44,75 \pm 0,35$ & $44,47 \pm 0,50$ & $44,53 \pm 0,41$ & $45,53 \pm 1,73$ & 0,41 & 0,33 & 0,12 \\
\hline $\mathrm{HB}(\mathrm{g} / \mathrm{dl})$ & $12,13 \pm 0,20 \mathrm{a}$ & $11,63 \pm 0,29 \mathrm{ab}$ & $13 \pm 0,23 \mathrm{bc}$ & $13,50 \pm 0,23 \mathrm{c}$ & 0,98 & $0,0001^{*}$ & $0,04 *$ \\
\hline HCM (uug) & $13,44 \pm 0,25 \mathrm{a}$ & $13,42 \pm 0,36 \mathrm{a}$ & $16,47 \pm 0,29 b$ & $16,60 \pm 0,29 b$ & 0,86 & $0,0001 *$ & 0,79 \\
\hline CHCM (\%) & $29,90 \pm 0,47 \mathrm{a}$ & $30,74 \pm 0,67 \mathrm{a}$ & $36,87 \pm 0,55 b$ & $36,60 \pm 0,55 b$ & 0,61 & $0,0001^{*}$ & 0,33 \\
\hline RGB (G/1) & $7,53 \pm 0,32 \mathrm{a}$ & $9,95 \pm 0,45 b$ & $9,72 \pm 0,37 b$ & $10,96 \pm 0,37 \mathrm{~b}$ & $0,0001 *$ & $0,0001 *$ & 0,12 \\
\hline Neutrófilos (\%) & $37,9 \pm 1,96 \mathrm{ab}$ & $37,20 \pm 2,77 b$ & $45,87 \pm 2,26 \mathrm{a}$ & $34,80 \pm 2,26 b$ & $0,01 *$ & 0,23 & $0,03 *$ \\
\hline Linfocitos (\%) & $54,65 \pm 1,85 \mathrm{a}$ & $54,80 \pm 2,61 \mathrm{a}$ & $35,5 \pm 2,13 b$ & $56,87 \pm 2,13 \mathrm{a}$ & $0,0001 *$ & $0,0003^{*}$ & $0,0001 *$ \\
\hline Monocitos (\%) & $2,65 \pm 0,45 \mathrm{a}$ & $2,40 \pm 0,64 a$ & $5 \pm 0,52 b$ & $3 \pm 0,52 \mathrm{a}$ & $0,04 *$ & $0,008 *$ & 0,11 \\
\hline Eosinófilos (\%) & $4,05 \pm 1 \mathrm{a}$ & $5,50 \pm 1,41 \mathrm{a}$ & $13,40 \pm 1,15 b$ & $5,27 \pm 1,15 \mathrm{a}$ & $0,007^{*}$ & $0,0003^{*}$ & $0,0002 *$ \\
\hline Basófilos (\%) & $0,20 \pm 0,08$ & $0,10 \pm 0,12$ & $0,20 \pm 0,09$ & $0,07 \pm 0,09$ & 0,86 & 0,23 & 0,87 \\
\hline CT (g/l) & $1,39 \pm 0,04 \mathrm{a}$ & $1,22 \pm 0,06 \mathrm{~b}$ & $1,58 \pm 0,05 \mathrm{c}$ & $1,17 \pm 0,05 b$ & $0,0001 *$ & 0,17 & $0,016^{*}$ \\
\hline TR (g/l) & $1,38 \pm 0,02 \mathrm{a}$ & $1,39 \pm 0,03 \mathrm{a}$ & $0,49 \pm 0,02 b$ & $0,38 \pm 0,02 \mathrm{c}$ & $0,05^{*}$ & $0,0001 *$ & $0,02 *$ \\
\hline C-HDL (g/l) & $0,66 \pm 0,04 \mathrm{a}$ & $0,51 \pm 0,04 b$ & $0,71 \pm 0,04 \mathrm{a}$ & $0,48 \pm 0,03 b$ & $0,0001 *$ & 0,77 & 0,34 \\
\hline C-LDL (g/l) & $0,63 \pm 0,03 \mathrm{a}$ & $0,52 \pm 0,03 b$ & $0,63 \pm 0,03 \mathrm{a}$ & $0,50 \pm 0,11 \mathrm{~b}$ & $0,0004^{*}$ & 0,73 & 0,81 \\
\hline PRT (g/dl) & $7,62 \pm 0,10 \mathrm{a}$ & $7,03 \pm 0,14 b$ & $6,14 \pm 0,11 \mathrm{c}$ & $5,79 \pm 0,11 \mathrm{c}$ & $0,0002^{*}$ & $0,0001 *$ & 0,31 \\
\hline ALB (g/dl) & $4,57 \pm 0,09 \mathrm{a}$ & $4,43 \pm 0,13 a$ & $3,33 \pm 0,11 b$ & $3,35 \pm 0,10 \mathrm{~b}$ & 0,58 & $0,0001 *$ & 0,47 \\
\hline GLOB (g/dl) & $3,05 \pm 0,12 \mathrm{a}$ & $2,60 \pm 0,17 \mathrm{ab}$ & $2,81 \pm 0,14 \mathrm{ab}$ & $2,44 \pm 0,14 \mathrm{a}$ & $0,006^{*}$ & 0,17 & 0,78 \\
\hline RAG & $1,60 \pm 0,10 \mathrm{ab}$ & $1,79 \pm 0,13 \mathrm{a}$ & $1,24 \pm 0,11 b$ & $1,40 \pm 0,11 \mathrm{ab}$ & 0,13 & $0,002 *$ & 0,88 \\
\hline Urea $(\mathrm{g} / \mathrm{l})$ & $0,70 \pm 0,01 \mathrm{a}$ & $0,67 \pm 0,02 \mathrm{a}$ & $0,58 \pm 0,01 b$ & $0,36 \pm 0,01 \mathrm{c}$ & $0,0001^{*}$ & $0,0001^{*}$ & $0,0001^{*}$ \\
\hline $\mathrm{CA}(\mathrm{mg} / \mathrm{dl})$ & $9,96 \pm 0,08 \mathrm{a}$ & $9,78 \pm 0,12 \mathrm{ab}$ & $9,47 \pm 0,10 \mathrm{~b}$ & $9,75 \pm 0,10 \mathrm{ab}$ & 0,60 & $0,02 *$ & $0,03 *$ \\
\hline FOS (mg/dl) & $5,38 \pm 0,29 a$ & $6,97 \pm 0,41 b$ & $5,43 \pm 0,35 \mathrm{a}$ & $6,18 \pm 0,36 b$ & $0,001^{*}$ & 0,30 & 0,24 \\
\hline MG (mg/dl) & $2,91 \pm 0,05 \mathrm{a}$ & $2,96 \pm 0,04 \mathrm{a}$ & $3,22 \pm 0,05 b$ & $3,38 \pm 0,05 b$ & 0,11 & $0,0001^{*}$ & 0,12 \\
\hline Glucosa (g/l) & $0,49 \pm 0,03 \mathrm{a}$ & $0,38 \pm 0,05 \mathrm{a}$ & $0,81 \pm 0,04 b$ & $0,92 \pm 0,04 \mathrm{~b}$ & 0,98 & $0,0001^{*}$ & $0,007 *$ \\
\hline LDH (UI/l) & $971 \pm 24 a$ & $1142 \pm 27 b$ & $456 \pm 22 \mathrm{c}$ & $506 \pm 23 \mathrm{c}$ & $0,0001 *$ & $0,0001 *$ & $0,02 *$ \\
\hline
\end{tabular}

Media \pm error estándar. Letras distintas indican diferencia (test de Tukey, significancia $\mathrm{p}<0,05$ ). local: localidad; interac: interacción; PV: peso vivo; PT: perímetro torácico; HTO: hematocrito; RGR: glóbulos rojos; HB: hemoglobina; VCM: volumen corpuscular medio; HCM: hemoglobina corpuscular media; CHCM: concentración de hemoglobina corpuscular media; RGB: glóbulos blancos; CT: colesterol total; TR: triglicéridos; C-HDL y C-LDL: colesterol ligado a lipoproteínas de alta y baja densidad respectivamente; PRT: proteínas totales; ALB: albúmina; GLOB: globulinas; RAG: relación albúmina-globulina; CA: calcio sérico; FOS: fósforo inorgánico; MG: magnesio; LDH: enzima lactato deshidrogenasa. 
Tabla 2. Efectos de la categoría (edad) de los animales, en las dos localidades estudiadas. Variables morfométricas, hemáticas y bioquímicas que resultaron con interacción edad x localidad.

\begin{tabular}{lcccccc}
\hline \multirow{2}{*}{ variable } & \multicolumn{3}{c}{ Itatí } & \multicolumn{3}{c}{ Empedrado } \\
\cline { 2 - 7 } & búfalas & bubillas & $\mathrm{p}$ & búfalas & bubillas & $\mathrm{p}$ \\
\hline PV (kg) & $553,10 \pm 10,64$ & $421,20 \pm 15,04$ & $0,0001^{*}$ & $598,33 \pm 2,30$ & $397,00 \pm 2,30$ & $0,0001^{*}$ \\
PT (cm) & $215,07 \pm 3,49$ & $188,30 \pm 4,94$ & $0,0001^{*}$ & $216,73 \pm 3,02$ & $174,07 \pm 3,02$ & $0,0001^{*}$ \\
HTO (\%) & $40,60 \pm 0,59$ & $37,40 \pm 0,83$ & $0,004^{*}$ & $36,8 \pm 0,43$ & $36,6 \pm 0,43$ & 0,66 \\
RGR (T/l) & $9,12 \pm 0,13$ & $8,38 \pm 0,18$ & $0,003^{*}$ & $8,13 \pm 0,15$ & $8,19 \pm 0,15$ & 0,80 \\
HB (g/dl) & $12,13 \pm 0,23$ & $11,63 \pm 0,32$ & 0,21 & $13,00 \pm 0,20$ & $13,50 \pm 0,20$ & 0,08 \\
Neutrófilos (\%) & $37,90 \pm 1,82$ & $37,20 \pm 2,58$ & 0,83 & $45,87 \pm 2,40$ & $34,80 \pm 2,40$ & $0,003^{*}$ \\
Linfocitos (\%) & $54,65 \pm 1,61$ & $54,80 \pm 2,28$ & 0,96 & $35,53 \pm 2,38$ & $56,87 \pm 2,38$ & $0,0001^{*}$ \\
Eosinófilos (\%) & $4,05 \pm 0,79$ & $5,50 \pm 1,12$ & 0,3 & $13,40 \pm 1,35$ & $5,27 \pm 1,35$ & $0,0002^{*}$ \\
CT (g/l) & $1,39 \pm 0,03$ & $1,22 \pm 0,04$ & $0,004^{*}$ & $1,58 \pm 0,06$ & $1,17 \pm 0,06$ & $0,0001^{*}$ \\
TR (g/l) & $1,38 \pm 0,02$ & $1,39 \pm 0,02$ & 0,57 & $0,49 \pm 0,03$ & $0,38 \pm 0,03$ & $0,02^{*}$ \\
Urea (g/l) & $0,70 \pm 0,02$ & $0,67 \pm 0,02$ & 0,41 & $0,58 \pm 0,01$ & $0,36 \pm 0,01$ & $0,0001^{*}$ \\
CA (mg/dl) & $9,96 \pm 0,10$ & $9,78 \pm 0,14$ & 0,31 & $9,47 \pm 0,08$ & $9,75 \pm 0,08$ & $0,02^{*}$ \\
Glucosa (g/l) & $0,49 \pm 0,04$ & $0,38 \pm 0,05$ & 0,09 & $0,81 \pm 0,03$ & $0,92 \pm 0,03$ & $0,03 *$ \\
LDH (UI/l) & $970,83 \pm 34,01$ & $1141,90 \pm 37,26$ & $0,003 *$ & $456,64 \pm 12,34$ & $506,08 \pm 12,8$ & $0,01 *$ \\
\hline
\end{tabular}

Media \pm error estándar. Significancia p <0,05. PV: peso vivo; PT: perímetro torácico; HTO: hematocrito; RGR: glóbulos rojos; HB: hemoglobina; CT: colesterol total; TR: triglicéridos; CA: calcio sérico; LDH: enzima lactato deshidrogenasa.

las hembras comience a los dos años de edad, con pesos aproximados de $400 \mathrm{~kg}^{4}$. Las bubillas que se evaluaron en nuestra experiencia, de ambas localidades, rondaron el peso óptimo citado por la bibliografía, como indicado para el inicio de la fase reproductiva.

El PT manifestó interacción edad por localidad $(\mathrm{p}=0,03)$. Este parámetro fue de $215,05 \pm 3,49$ y $188,30 \pm 4,94 \mathrm{~cm}$ para las búfalas y bubillas de Itatí $(\mathrm{p}=0,0001)$ y de $216,73 \pm 3,02$ y $174,07 \pm 3,02 \mathrm{~cm}$ para las mismas categorías de Empedrado. No se registraron diferencias de PT entre las hembras adultas de ambas localidades; las bubillas de Itatí ostentaron mayores PT $(188,30 \pm 3,93 \mathrm{~cm})$ que las de Empedrado $(174,07 \pm 3,02$ $\mathrm{cm})$.
Otros autores trabajaron con un rodeo bubalino lechero mestizo de raza Murrah, machos y hembras, con el fin de evaluar la posible correlación entre el peso vivo y perímetro torácico ${ }^{2}$. A través del estudio, demostraron que este último puede ser utilizado para determinar el peso vivo de los animales, debiendo siempre considerarse al sexo, como un factor de variación.

Los parámetros hemáticos HTO, RGR y HB mostraron interacción positiva entre los efectos de edad y localidad ( $\mathrm{p}=0,004 ; \mathrm{p}=0,01$ y 0,04 respectivamente). Las búfalas adultas de Itatí, revelaron significativamente mayores valores de $\mathrm{HTO}(\mathrm{p}=0,004)$ y $\mathrm{RGR}(\mathrm{p}=0,003)$ que las bubillas de la misma localidad, resultado que no fue observado en los animales de Empedrado (Tabla 2). Las búfalas de Itatí revelaron mayores valores

Tabla 3. Efectos de la localidad (lugar de residencia) sobre la categoría de los animales. Variables morfométricas, hemáticas y bioquímicas que resultaron con interacción edad por localidad.

\begin{tabular}{|c|c|c|c|c|c|c|}
\hline \multirow{2}{*}{ variable } & \multicolumn{3}{|c|}{ búfalas } & \multicolumn{3}{|c|}{ bubillas } \\
\hline & Itatí & Empedrado & $\mathrm{p}$ & Itatí & Empedrado & $\mathrm{p}$ \\
\hline$\overline{\mathrm{PV}(\mathrm{kg})}$ & $553,10 \pm 9,26$ & $598,33 \pm 10,60$ & $0,003^{*}$ & $421,20 \pm 6,26$ & $397,00 \pm 5,11$ & $0,007 *$ \\
\hline PT (cm) & $215,05 \pm 3,28$ & $216,73 \pm 3,78$ & 0,74 & $188,30 \pm 3,93$ & $174,07 \pm 3,21$ & $0,01 *$ \\
\hline HTO (\%) & $40,60 \pm 0,48$ & $36,60 \pm 0,55$ & $0,0001 *$ & $37,40 \pm 0,73$ & $36,87 \pm 0,059$ & 0,58 \\
\hline RGR (T/1) & $9,12 \pm 0,14$ & $8,13 \pm 0,16$ & $0,0001 *$ & $8,38 \pm 0,17$ & $8,19 \pm 0,14$ & 0,4 \\
\hline $\mathrm{HB}(\mathrm{g} / \mathrm{dl})$ & $12,13 \pm 0,22$ & $13,00 \pm 0,25$ & $0,01 *$ & $11,63 \pm 0,25$ & $13,50 \pm 0,20$ & $0,0001^{*}$ \\
\hline Neutrófilos (\%) & $37,90 \pm 2,00$ & $45,87 \pm 2,30$ & $0,01 *$ & $37,20 \pm 2,68$ & $34,80 \pm 2,19$ & 0,49 \\
\hline Linfocitos $(\%)$ & $54,65 \pm 1,84$ & $35,53 \pm 2,13$ & $0,0001 *$ & $54,80 \pm 2,62$ & $56,87 \pm 2,14$ & 0,55 \\
\hline Eosinófilos (\%) & $4,05 \pm 0,79$ & $13,40 \pm 1,35$ & $0,0001 *$ & $5,50 \pm 1,11$ & $5,27 \pm 0,91$ & 0,87 \\
\hline $\mathrm{CT}(\mathrm{g} / \mathrm{l})$ & $1,39 \pm 0,05$ & $1,58 \pm 0,05$ & $0,01^{*}$ & $1,22 \pm 0,04$ & $1,17 \pm 0,04$ & 0,33 \\
\hline TR (g/l) & $1,38 \pm 0,02$ & $0,49 \pm 0,02$ & $0,0001 *$ & $1,39 \pm 0,03$ & $0,38 \pm 0,03$ & $0,0001 *$ \\
\hline Urea $(g / 1)$ & $0,70 \pm 0,01$ & $0,58 \pm 0,02$ & $0,0001 *$ & $0,67 \pm 0,01$ & $0,36 \pm 0,01$ & $0,0001 *$ \\
\hline $\mathrm{CA}(\mathrm{mg} / \mathrm{dl})$ & $9,96 \pm 0,09$ & $9,47 \pm 0,11$ & $0,002^{*}$ & $9,78 \pm 0,09$ & $9,75 \pm 0,08$ & 0,83 \\
\hline Glucosa (g/l) & $0,49 \pm 0,03$ & $0,81 \pm 0,04$ & $0,0001^{*}$ & $0,38 \pm 0,04$ & $0,92 \pm 0,03$ & $0,0001 *$ \\
\hline LDH (UI/l) & $970,83 \pm 21,71$ & $456,64 \pm 20,10$ & $0,0001 *$ & $1141,90 \pm 30,49$ & $506,08 \pm 26,74$ & $0,0001 *$ \\
\hline
\end{tabular}

Media \pm error estándar. Significancia p $<0,05$. PV: peso vivo; PT: perímetro torácico; HTO: hematocrito; RGR: glóbulos rojos; HB: hemoglobina; CT: colesterol total; TR: triglicéridos; CA: calcio sérico; LDH: enzima lactato deshidrogenasa. 
de HTO y RGR que las de Empedrado ( $\mathrm{p}=0,0001$ para ambos parámetros), mientras que estas diferencias no se observaron en la categoría bubillas (Tabla 3).

En otra investigación se obtuvieron niveles de RGR de $7,55 \pm 1,02$ y $6,53 \pm 0,81 \mathrm{~T} / 1$; HTO de $34,00 \pm 5,90$ y $33,10 \pm 4,00 \%$ y HB de $10,70 \pm 1,30$ y $10,80 \pm 1,41 \mathrm{~g} / \mathrm{dl}$, para grupos de bubalinos de raza Murrah de 9 meses a 2 años y mayores de 2 años, de la región del Amazonas ${ }^{11}$. Asímismo, estos autores observaron que los búfalos más jóvenes (de 2 a 8 meses) tuvieron valores más altos de HTO $(39,62 \pm 4,01 \%)$, HB $(12,81 \pm 1,53 \mathrm{~g} / \mathrm{dl})$ y RGR $(9,80 \pm 1,22(\mathrm{~T} / \mathrm{l})$. Estos aumentos podrían atribuirse a la expansión del volumen sanguíneo, producto de una mayor demanda de eritrocitos y un incremento de la actividad hematopoyética por la que atraviesan estos animales durante la fase de crecimiento rápido, en los primeros meses de vida ${ }^{13}$.

Otras experiencias realizadas con búfalos de agua, hacen referencia que los valores de RGR, HTO y HB son más altos en los animales jóvenes, observándose una significativa disminución a mediada avanza la edad ${ }^{28}$. Para bubalinos de raza Murrah del estado de São Paulo, de 7 meses a 1 año y de 1 a 5 años, estudios reportaron RGR de 7,9 $\pm 1,2$ y de $6,7 \pm 1,7 \mathrm{~T} / 1$, HB de $12,0 \pm 1,2$ y $11,7 \pm 1,8, \mathrm{~g} / \mathrm{dl}$ y HTO de $33,8 \pm 3,2$ y de $34,4 \pm 3,4 \%$, respectivamente ${ }^{16}$. El RGR fue menor en la especie bubalina, en relación a la bovina, en los primeros 30 días de edad ${ }^{9}$.

Los resultados obtenidos en nuestra experiencia muestran un comportamiento distinto, ya que los niveles de estos parámetros hematológicos fueron mayores en las búfalas adultas. En concordancia con otro estudio donde se evaluaron hembras de distintas edades ${ }^{10}$, en bubillas de 12 meses se obtuvieron HTO cercanos al 33\%, mientras que en búfalas de 30 meses, el valor hallado fue de $37 \%$. Otros autores coinciden en estos índices, al explorar rodeos de búfalas mestizas Murrah de aproximadamente 18 meses de edad, en Maracaibo, Venezuela ${ }^{3}$.

En la región de Magdalena, Colombia, se hallaron niveles de HTO promedio de $34 \%$, al evaluar grupos de búfalos de entre 12 y 36 meses, sin distinción de sexo ${ }^{23}$. En tanto, en el estado de Táchira, Venezuela, al analizar las variables hematológicas de 105 búfalos jóvenes de las razas Murrah, Nilli Ravi y Jafarabadi, de hasta 30 meses de edad, no se encontraron diferencias significativas entre sexos salvo en los valores de HTO, que fue mayor en las hembras (37\%) en relación a los machos (35\%). En la misma investigación, los animales agrupados según sus pesos hasta $150 \mathrm{~kg}$, de 150-350 $\mathrm{kg}$ y mayores de $350 \mathrm{~kg}$, surgió que las diferencias se acentuaban entre los animales del primer y segundo grupo, con respecto al tercero, mostrando este último, descensos de RGR y HTO, así como aumentos de HB, HCM y CHCM, sin variaciones del VCM ${ }^{19}$.

En nuestro trabajo, el VCM no se vio influenciado por los efectos de la edad y el lugar de residencia, en tanto que HB, HCM, CHCM difirieron en los animales de las dos localidades. Mayores valores de estos últi- mos parámetros se registraron en el establecimiento próximo a Empedrado (Tabla 1). La HB reveló interacción significativa $(\mathrm{p}=0,004)$. La edad no influenció sobre este indicador hemático, pero si la localidad. Las búfalas $(13 \pm 0,25 \mathrm{~g} / \mathrm{dl}, \mathrm{p}=0,01)$ y bubillas $13,50 \pm 0,20 \mathrm{~g} /$ $\mathrm{dl}, \mathrm{p}=0,0001)$ de Empedrado revelaron mayores valores de HB que las hembras adultas $(12,13 \pm 0,22 \mathrm{~g} / \mathrm{dl})$ y jóvenes $(11,63 \pm 0,25 \mathrm{~g} / \mathrm{dl})$ de Itatí (Tabla 3$)$.

En un trabajo se halló que los niveles de HB fueron menores en los animales de 12 meses, en relación al incremento observado en los búfalos de 24 meses de edad ${ }^{16}$. En concordancia, en otra investigación, se observó que el valor de HB fue menor en los animales de 3 a 4 años, en relación con los de 5 a 6 años ${ }^{18}$.

Otros autores citan disminuciones de HB, RGR, HTO y VCM con el avance de edad, (en grupos etarios de hembras bubalinas, desde el nacimiento hasta los 72 meses de vida), no así de los valores de HCM y CHCM, que aumentaron paralelamente a la edad. Asímismo, no se encontraron diferencias estadísticamente significativas en las variables hematológicas, en relación al factor racial de los animales, al trabajar con hembras de raza Murrah, Jafarabadi y Mediterránea, en la región del Valle de la Rivera, San Pablo, Brasil ${ }^{15}$. La concentración de HB fue mayor en búfalos que en bovinos, en animales hasta los 45 días de vida ${ }^{9}$, y también hubo influencia del sexo en el valor de $\mathrm{VCM}^{14}$. Las hembras $(49,29 \pm 2,5 \mathrm{fl})$ presentaron mayores valores de VCM que los machos (43,28 $\pm 1,2 \mathrm{fl})$. Un autor propone que la disminución del VCM se deba probablemente a un menor volumen de los eritrocitos como consecuencia de un menor contenido de $\mathrm{HB}^{20}$.

El RGB se presentó significativamente más alto en la categoría bubillas, y fue mayor en los ejemplares de la localidad de Empedrado (Tabla 1). Los porcentajes de neutrófilos, monocitos y eosinófilos revelaron interacción de los efectos edad por lugar de origen. Las búfalas de Empedrado presentaron significativamente porcentuales más elevados de estos glóbulos blancos que las bubillas de igual localidad, efecto que no fue registrado en Itatí (Tabla 2).

En dicha localidad, las hembras adultas mostraron mayores porcentajes de linfocitos ( $p=0,0001)$, mientras que en las de Empedrado fueron mayores los neutrófilos $(p=0,01)$ y eosinófilos $(p=0,0001)$. Las bubillas de ambas localidades no revelaron diferencias entre estas variables leucocitarias (Tabla 3). Los efectos analizados en la experiencia no influyeron sobre los porcentajes de monocitos y basófilos (Tabla 1).

Al evaluar búfalos de 12 y 24 meses de edad se obtuvieron valores de glóbulos blancos de 11,9 G/1 para el grupo más joven y de 14,1 G/1 para los adultos ${ }^{12}$. Búfalos de Colombia revelaron recuentos diferenciales con $22-26 \%$ de neutrófilos, $66-69 \%$ de linfocitos, $4-6 \%$ de monocitos, $1-2 \%$ de eosinófilos y $0,2-0,5 \%$ de basófilos ${ }^{23}$. Otros autores citan valores de recuentos diferenciales muy similares a los aquí hallados (neutrófilos $30,92 \%$, linfocitos $65 \%$, monocitos 3,23\% y eosinófilos 
$0,92 \%)$, no así para el recuento de leucocitos totales, que resultó menor $(10,24 \mathrm{G} / 1)^{10}$.

En búfalos de la Amazonia se constató influencia de la edad en el RGB, obteniendo valores significativamente mayores en búfalos de 2 a 8 meses $(15,95 \pm 3,70$ $\mathrm{G} / \mathrm{l})$, mientras que para animales de 9 meses a 2 años y mayores a 2 años, el RGB fue de 12,41 $\pm 2,70 \mathrm{G} / 1$ y $9,20 \pm 2,71 \mathrm{G} / 1$, respectivamente ${ }^{11}$. Estos mismo autores señalan que la relación neutrófilos/linfocitos fue inferior a 1 en todos los grupos etarios que consideraron en su trabajo, revelando el predominio de linfocitos, por sobre los neutrófilos, circunstancia que coincide con lo citado en diferentes trabajos ${ }^{23}$.

En nuestra experiencia, las búfalas y bubillas de la localidad de Itatí, mostraron una relación neutrófilos/linfocitos de 0,69 y 0,68, respectivamente. Para las hembras jóvenes de Empedrado, la relación fue de 0,68; mientras que para las adultas fue de 1,29. Otros autores encontraron una prevalencia de neutrófilos sobre linfocitos, en bucerros de hasta 8 días, diferencia que se atribuiría a la edad ${ }^{10}$.

Un estudio sobre el leucograma de búfalos aseveró que no existirían diferencias atribuibles a las razas (Mediterránea, Jafarabadi y Murrah), pero sí a la edad, ya que al incrementarse provocaría la elevación de neutrófilos y eosinófilos, con disminución del RGB y los linfocitos ${ }^{29}$. Estas observaciones concuerdan con otras citas que indican que tales diferencias serían más significativas en la especie bubalina que en la bovina, en animales dentro de los primeros 45 días de vida ${ }^{9}$.

En un trabajo se hace referencia a un recuento de eosinófilos más alto en los animales de los rangos etarios mayores ( 9 meses a 2 años versus mayores de 2 años) ${ }^{11}$. Este incremento de la eosinofilia acorde a la edad, puede atribuirse a una mayor exposición de los animales a los parásitos gastrointestinales ${ }^{20}$. La influencia de la edad de los bubalinos sobre el número de eosinófilos fue observada también por otros autores ${ }^{12} \mathrm{y}$ concuerda con los resultados obtenidos en nuestro trabajo. El comportamiento de la eosinofilia también es citado en la especie bovina ${ }^{9}$.

La categoría bubilla evidenció valores de PRT significativamente más bajos $(\mathrm{p}=0,0002)$ que los observados en la categoría búfalas. Este parámetro fue mayor en las hembras de la localidad de Itatí $(p=0,0001)$ (Tabla 1). Evaluando un grupo de búfalos de 12 a 36 meses, sin distinción de sexo, se registró una tendencia ascendente a medida que avanzaba la edad ${ }^{23}$. Otros describen que la concentración de PRT es influenciada por la faja etaria de los animales (de 2 a 8 meses $8,05 \pm 0,51$ $\mathrm{g} / \mathrm{dl}$; de 9 meses a 2 años $8,3 \pm 0,90 \mathrm{~g} / \mathrm{dl}$ y mayores a 2 años $9,2 \pm 1,74 \mathrm{~g} / \mathrm{dl}$ ) siendo menor en los animales más jóvenes ${ }^{11,15}$.

En una experiencia en la que se muestrearon 127 búfalas de entre 1 y 2 años de edad, de varias granjas de los valles de Egipto, se reportó un intervalo de referencia de 5,60 a 8,10 g/dl para los valores de PRT (media de $6,80 \pm 0,69 \mathrm{~g} / \mathrm{dl})^{1}$. Otros investigadores citan niveles de 6,70 a $7,40 \mathrm{~g} / \mathrm{dl}$ (media 7,10 $\pm 0,18 \mathrm{~g} / \mathrm{dl})^{21}$.
Investigadores han postulado que el incremento de los valores de PRT, en paralelo a la edad, también se observa en la especie bovina ${ }^{9}$. La concentración plasmática de proteínas, incluyendo a las globulinas, aumenta rápidamente horas después del consumo del calostro debido a la absorción intestinal de $\gamma$-globulinas y $\beta$-globulinas ${ }^{21}$. En hembras bubalinas de raza Murrah en lactación, los niveles de PRT son en general más bajos en la temporada de verano que durante el invierno ${ }^{30}$. En bucerros sometidos a estrés térmico, los valores séricos de PRT disminuyen significativamente ${ }^{17}$.

Los valores de ALB no se vieron afectados por la edad de los animales, pero sí revelaron modificaciones significativas $(\mathrm{p}=0,0001)$ debidas a la localidad. Mayores concentraciones de esta variable se observaron en las búfalas de Itatí (Tabla 1). Elevados niveles de ALB sérica son citados para la especie bubalina, en relación a la bovina ${ }^{9}$.

El valor promedio de albuminemia reportado para búfalas de 1 a 2 años, fue de 3,20 $\pm 0,47 \mathrm{~g} / \mathrm{dl}$ (2,4-4,07 $\mathrm{g} / \mathrm{dl})$ y de GLOB: $3,50 \pm 0,65 \mathrm{~g} / \mathrm{dl}(2,34-5 \mathrm{~g} / \mathrm{dl})^{1}$. Otros autores hacen referencia a rangos de ALB de 3,03 a $3,50 \mathrm{~g} / \mathrm{dl}$ (media 3,92 $\pm 0,13 \mathrm{~g} / \mathrm{dl}$ ) y de GLOB de 3 a 3,48 $\mathrm{g} / \mathrm{dl}$ (media 3,24 $\pm 0,24 \mathrm{~g} / \mathrm{dl}$ ) ${ }^{21}$. En nuestro trabajo, las GLOB fueron mayores en hembras adultas, con relación a las bubillas, en ambas localidades (Tabla 1).

La RAG fue más elevada en las búfalas de Itatí que en las de Empedrado ( $p=0,002)$. La edad no influyó sobre esta variable (Tabla 1). Los resultados obtenidos en nuestra experiencia, fueron mayores a los reportados en otros trabajos (valor medio $0,96 \pm 0,27$; intervalo de $0,56-1,67)^{1}$.

La urea reveló una significativa interacción $(\mathrm{p}=0,0001)$ entre los efectos de la edad y la localidad (Tabla 1). Este indicador nitrogenado se mostró más elevado $(p=0,0001)$ en las búfalas mayores que en las jóvenes de la localidad de Empedrado, no así en las de Itatí (Tabla 2). Asimismo, las hembras jóvenes $(p=0,0001)$ y adultas $(p=0,0001)$ de Itatí, ostentaron valores superiores que las de Empedrado (Tabla 3).

Diferentes trabajos mencionan valores de uremia de $0,21 \pm 0,14 \mathrm{~g} / 1$ para bucerros de 2 a 8 meses, de $0,30 \pm 0,17 \mathrm{~g} / 1$ para animales de 9 meses a 2 años y de $0,29 \pm 0,07 \mathrm{~g} / 1$ para mayores a 2 años ${ }^{11}$, valores medios de $0,4 \pm 0,09 \mathrm{~g} / 1(0,21-0,59)$ para bubillas de 1 a 2 años ${ }^{1}$ o niveles de 0,2 a $0,3 \mathrm{~g} / 1$ para este mismo parámetro ${ }^{21}$.

Las concentraciones de urea sérica fueron mayores en la especie bubalina, que en la bovina ${ }^{9}$. Bucerros sometidos a condiciones de estrés, elevan significativamente sus niveles de uremia ${ }^{17}$. La exposición aguda al calor $\left(33-43^{\circ} \mathrm{C}\right.$ y $40-60 \%$ de humedad relativa) no generó cambios manifiestos de los valores de urea plasmática, tanto en búfalos jóvenes (de 6 meses) como adultos (mayores de 12 meses) ${ }^{25}$.

La glucemia mostró una significativa interacción $(p=0,02)$ entre la influencia de la edad y la localidad (Tabla 1). Esta variable se mostró más elevada en los animales de Empedrado (Tabla 3). Asimismo, las bubillas de este lugar presentaron los mayores valores de 
glucosa sérica $(0,92 \pm 0,03 \mathrm{~g} / \mathrm{l})$ que las búfalas adultas de la misma localidad $(0,81 \pm 0,03 \mathrm{~g} / \mathrm{l})$ y que las hembras jóvenes $(0,38 \pm 0,05 \mathrm{~g} / \mathrm{l})$ y adultas $(0,49 \pm 0,04)$ de la localidad de Itatí (Tabla 2).

Un estudio refiere que los niveles de glucosa sérica disminuyen con la edad y que este parámetro fue menor en búfalos que en bovinos ${ }^{9}$. En trabajos con bubillas de raza Murrah, se observó que aquéllas que recibieron altos tenores energéticos, presentaron mayores concentraciones circulantes de sustratos metabólicos como glucosa, en relación a las que recibieron dietas de bajo contenido calórico ${ }^{6}$. Otros citan valores medios de glucemia de $0,63 \pm 0,14 \mathrm{~g} / 1$ (intervalo $0,35-0,92$ ) para bubillas de 1 a 2 años de edad ${ }^{1}$ o niveles de 0,45 a 0,75 $\mathrm{g} / 1$ (media $0,57 \pm 0,06 \mathrm{~g} / \mathrm{l}$ ) ${ }^{21}$. La exposición aguda al calor $\left(33-43^{\circ} \mathrm{C}\right.$ y $40-60 \%$ de humedad relativa) a bucerros de meses, puede ocasionar incrementos de glucosa en plasma de hasta el $58 \%{ }^{25}$.

Los valores de CT y TR revelaron interacción entre los efectos de la edad y el lugar de residencia de los animales (Tabla 1). Las hembras adultas exhibieron mayores niveles de CT que las hembras jóvenes de ambas localidades (Tabla 2). Las búfalas de Itatí mostraron menores valores de CT $(1,39 \pm 0,05 \mathrm{~g} / \mathrm{l})$ que las de Empedrado (1,58 $\pm 0,05 \mathrm{~g} / \mathrm{l})$ (Tabla 3).

Los niveles de TR de las hembras de Itatí no revelaron diferencias de acuerdo a la edad, no así las de Empedrado, donde las búfalas adultas mostraron $(\mathrm{p}=0,02)$ mayores concentraciones que las bubillas (Tabla 2). Los animales jóvenes de Itatí presentaron niveles de TR $(1,39 \pm 0,03 \mathrm{~g} / \mathrm{l})$ significativamente más elevados $(\mathrm{p}=0,0001)$ que las del establecimiento próximo a Empedrado $(0,38 \pm 0,03 \mathrm{~g} / \mathrm{l})$, hecho que se repitió en los animales mayores $(\mathrm{p}=0,0001)$ (Tabla 3 ).

La bibliografía consultada hace referencia a valores medios de CT de $0,56 \pm 0,10 \mathrm{~g} / 1$; en un rango de 0,35 a $0,78 \mathrm{~g} / 1$ para hembras bubalinas de 12 a 24 meses de distintas granjas de Egipto ${ }^{1} \mathrm{y}$ otros autores citan niveles de 0,8 a $1,20 \mathrm{~g} / \mathrm{l}^{21}$. Al evaluar el efecto de las estaciones del año, se observaron concentraciones de colesterol sanguíneo más bajas durante el verano que durante la temporada de invierno, durante el período de lactancia de hembras bubalinas de la raza Murrah ${ }^{30}$. Esto indicaría que los valores de CT disminuyen con el aumento de la temperatura ambiente ${ }^{17}$.

Los resultados obtenidos por otros autores para TR variaron entre $0,27 \pm 0,11 \mathrm{~g} / 1(0,04-0,49){ }^{1} \mathrm{y}$ de $0,001 \mathrm{a}$ $0,14 \mathrm{~g} / \mathrm{l}^{21}$. Estos valores fueron similares a los observados en nuestro trabajo, en las búfalas y bubillas de la localidad de Empedrado, no así para las de Itatí, donde los niveles hallados fueron significativamente más elevados (Tabla 3). En experiencias donde se apreciaron los efectos de la restricción alimentaria, se observaron marcados incrementos de los TR, como consecuencia, posiblemente, de la mayor lipólisis del tejido graso de reserva ${ }^{27}$.

Las concentraciones séricas de colesterol-HDL y de colesterol-LDL se mostraron más elevadas en las búfalas que en las bubillas ( $p=0,0001$ y $p=0,0004$, res- pectivamente). No se observaron efectos de la localidad sobre estos indicadores lipídicos (Tabla 1). Algunos autores citan valores de C-HDL de 0,32 $\pm 0,10 \mathrm{~g} / 1$, con un intervalo de referencia de 0,12 a $0,52 \mathrm{~g} / 1$ y de C-LDL de $0,18 \pm 0,06 \mathrm{~g} / 1$ (rango 0,04 a $0,31 \mathrm{~g} / \mathrm{l}$ ) para hembras bubalinas de 12 a 24 meses ${ }^{1}$.

Los valores de CA sérico revelaron una significativa interacción entre los efectos de la edad y la localidad ( $p=0,03$ ). Las bubillas de Empedrado mostraron una calcemia más elevada que las búfalas adultas del mismo lugar $(\mathrm{p}=0,02)$, hecho que no se observó en la localidad de Itatí $(\mathrm{p}=0,3)$ (Tabla 2). Los animales de Itatí (búfalas $9,96 \pm 0,09 \mathrm{mg} / \mathrm{dl}$ y bubillas $9,78 \pm 0,09 \mathrm{mg} / \mathrm{dl}$ ) presentaron mayores niveles de calcio que los de Empedrado (búfalas $9,47 \pm 0,11 \mathrm{mg} / \mathrm{dl}$ y bubillas $9,75 \pm 0,08$ mg/dl) (Tabla 3). En búfalas de 1 a 2 años se reportaron valores medios de CA de $10,29 \pm 1,11 \mathrm{mg} / \mathrm{dl}(8,11$ $12,46)^{1}$, y $11,08 \pm 0,67 \mathrm{mg} / \mathrm{dl}(9,70-12,4)^{21}$.

La FOS no reveló variaciones por efecto de la localidad pero si por la edad. Las bubillas de ambas localidades mostraron niveles más altos de fósforo, que las hembras adultas (Tabla 1). Los valores medios observados en nuestra experiencia para las búfalas jóvenes, son muy similares a los reportados en otros trabajos, con medias de 6,57 $\pm 0,75 \mathrm{mg} / \mathrm{dl}$; rango de 4,39-7,85 para animales de 1 a 2 años de edad ${ }^{1}$ y 5,60-6,50 mg/dl ${ }^{21}$.

Los valores de MG sérico fueron significativamente mayores $(p=0,0001)$ en las hembras de Empedrado (búfalas $3,22 \pm 0,05 \mathrm{mg} / \mathrm{dl}$ y bubillas $3,38 \pm 0,05 \mathrm{mg} / \mathrm{dl}$ ) que en las de Itatí (búfalas $2,91 \pm 0,05 \mathrm{mg} / \mathrm{dl}$ y bubillas $2,96 \pm 0,04 \mathrm{mg} / \mathrm{dl})$. La bibliografía consultada hace referencia a valores de magnesemia, para bubillas jóvenes, de $2,69 \pm 0,46 \mathrm{mg} / \mathrm{dl}(1,78-3,59){ }^{1} \mathrm{y}$ de $2,05 \pm 0,25 \mathrm{mg} / \mathrm{dl}$ $(1,80-2,30)^{21}$. Otros autores indican que los niveles séricos de CA y FOS son similares entre bovinos y búfalos, no así los del MG, que se presentan más elevados en la especie bubalina ${ }^{9}$.

La enzima LDH evidenció interacción entre la influencia de la edad y la localidad ( $p=0,02)$. Las bubillas mostraron mayores niveles sanguíneos de lactato dehidrogenasa que las búfalas adultas, en ambas localidades (Tabla 2). Las hembras de Itatí presentaron valores más altos de LDH (búfalas 970,83 $\pm 21,71 \mathrm{UI} / 1$ y bubillas 1141,90 $\pm 30,49 \mathrm{UI} / 1$ ) que las de Empedrado (búfalas $456,64 \pm 20,10 \mathrm{UI} / 1$ y bubillas 506,08 $\pm 26,74 \mathrm{UI} / \mathrm{l}$ ) (Tabla $3)$.

La bibliografía consultada reveló escasas referencias de la enzima LDH en la especie bubalina. Algunos autores señalan valores de $546,18 \pm 232,71 \mathrm{UI} / 1$, con un intervalo de referencia de 186,72 a 917,43 UI/l para búfalas de 12 a 24 meses ${ }^{1}$ o niveles medios de $1061 \pm 222$ (692 a $1445 \mathrm{UI} / \mathrm{l})^{21}$. Los valores enzimáticos obtenidos en nuestra experiencia están dentro de los rangos citados.

Concluyendo, surge que la obtención de intervalos de referencia regionales para valores de laboratorio de esta especie animal, asume importancia para optimizar el diagnóstico de enfermedades, como así también para valorar los indicadores nutricionales sanguíneos 
que, en conjunto con la medición de parámetros morfométricos, constituyen herramientas útiles para evaluar la eficacia de los emprendimientos productivos.

Agradecimientos. A la empresa Wiener Lab (Rosario, Argentina), por proveer los reactivos necesarios para la realización de los análisis.

\section{REFERENCIAS}

1. Abd Ellah MR, Hamed MI, Ibrahim DR, Rateb HZ. 2014. Serum biochemical and haematological reference intervals for water buffalo (Bubalus bubalis) heifers. J South Afr Vet Ass 85: 962-970.

2. Alcântara DC, Araújo CV, Bittencourt RH, Rodrigues FE, Colino EC, Silva MC. 2008. Estudo preliminar da correlação entre o perímetro torácico e o peso corporal de búfalos leiteiros mestiços Murrah (Bubalus bubalis). Anais $35^{\circ}$ Congreso Brasilero de Medicina Veterinaria. Gramado, Brasil, Resumen cd R0302.

3. Azuaje R, Sánchez D. 2009. Valores hematológicos en búfalos (Bubalis bubalis) y bovinos (Bos taurus-indicus) en fincas del sur del lago de Maracaibo. Mund Pec Rev 3: 168-218.

4. Baruselli PS. 2007. Reprodução em búfalos. On line: http://www.fmvz.usp.br/menu /sitebra11.html $>21$.

5. Bavera GA. 2011. Razas bovinas y bufalinas de la Argentina, Ed. Imberti, Rio Cuarto (Córdoba, Argentina), 261 p.

6. Campanile G et al. 2011. Growth, metabolic status and ovarian function in buffalo (Bubalus bubalis) heifers fed a low energy or high-energy diet. Anim Reprod Sci 22: 74-81.

7. Damasceno FA, Viana JM, Tinôco IF, Gomes RC, Schiassi L. 2010. Adaptação de bubalinos ao ambiente tropical. Nutr Rev Eletr 125: 1370-1381.

8. Das GK, Khan FA. 2010. Summer anoestrus in buffalo. A Review. Reprod Dom Anim 45: 483-494.

9. Fagliari JJ, Santana AE, Lucas FA, Campos PR, Curi PR. 1998. Constituintes sangüíneos de bovinos recém-nascidos das raças Nelore (Bos indicus) e Holandesa (Bos taurus) e de bubalinos (Bubalus bubalis) da raça Murrah. Arq Bras Med Vet Zootec 50: 253-262.

10. Ferrer JM, Árraga CM, Barboza M. 2000. Caracterización hematológica de Bubalus bubalis por sexo y edad. Rev Cient FCV-LUZ 10: 508-514.

11. Fontes DG et al. 2014. Perfil hematológico e bioquímico de búfalos (Bubalus bubalis) na Amazônia Oriental. Pes Vet Bras 34: 57-63.

12. França $\mathbf{R}$ et al. 2011. Valores hematológicos de búfalos em diferentes faixas etárias criados na região central do Rio Grande do Sul. Rev Bras Cien Vet 1: 51-54.

13. Freitas ML, Pinheiro DM, Ginani F, Barreto MP, Barboza CA. 2012. Influência do envelhecimento no rendimento in vitro de células-tronco mesenquimais da medula óssea de camundongos. J Health Sci Inst 30: 103-106.

14. Gerometta J. 2015. La cría de búfalos se consolida en la región con ventajas competitivas. On Line: www.diarionorte. com/article/125668.

15. Gomes V, Madureira KM, Blagitz MG, Galdino J, Vantim G, Benesi FJ. 2010. Valores de referência e in- fluência do etário sobre os parâmetros bioquímicos utilizados para avaliação da função hepática de bubalinos hígidos da raça Murrah. Ars Vet 26: 128-131.

16. Gomes V et al. 2010. Valores de referencia e influencia da idade no eritrograma de bubalinos da raça Murrah. Pesq Vet Bras 30: 301-304.

17. Habeeb AA, Fatma FI, Osman SF. 2007. Detection of heat adaptability using heat shock proteins and some hormones in Egyptian buffalo calves. Egypt J Appl Sci 22: 28-53.

18. Hernandez A, Romero O, Montiel N, Nava H, Cahuao N. 2005. Determinación de valores de referencia hematológicos en búfalas (Bubalus bubalis) preparto y postparto en una unidad de producción en el sur del lago de Maracaibo, Venezuela. Rev Cient FCV-LUZ 15: 119-124.

19. Iglesia LM, Azuaje KK, Sanchez F, Ramirez AD. 1990. Observaciones hematológicas en búfalos de água (Bubalus bubalis) parentemente sanos em el Occidente de Venezuela. Rev Cient FCV-LUZ 9: 524-531.

20. Jain NC. 1993. Essentials of Veterinary Hematology, Lea \& Febiger, Philadelphia, $1344 \mathrm{p}$.

21. Kaneko JJ, Harvey JW, Bruss LM. 2008. Clinical Biochemistry of Domestic Animals, $6^{\circ}$ ed., Academic Press, San Diego (USA), $932 \mathrm{p}$.

22. Klinkon M, Jezek J. 2012. Values of blood variable in calves. A birds-eye views of veterinary medicine. On line: http://www.intechopen.com/books/a-bird-s-eye-view-ofveterinary-medicine/values-of-blood-variables-in-calves.

23. Londoño R, Sánchez M, Prada G. 2012. Parámetros fisiológicos y valores hematológicos normales en búfalos (Bubalus bubalis) del Magdalena Medio colombiano. Rev Med Vet 23: 51-64.

24. Nascimento C, Carvalho LO. 1993. Criação de Búfalos. Alimentação, Manejo, Melhoramento e Instalações. Anais EMBRAPA, Brasilia, 403 p.

25. Nessim MG. 2004. Heat-induced biological changes as heat tolerance indices related to growth performance in buffaloes. Ph Doctoral Thesis, Faculty of Agriculture, Ain Shams University, Cairo, Egypt.

26. Perera BM. 2010. Reproductive cycles of buffalo. Anim Reprod Sci. On line: www.animalreproductionscience. com/doi:10.1016/j.anireprosci.2010.08.022.

27. Rodríguez EJ, Carande VG, Rodríguez VA. 1985. Efectos de la restricción y la realimentación sobre la concentración de metabolitos sanguíneos. Rev Arg Prod Anim 5: $1-12$.

28. Silva MB, D’Angelino JL, Araujo WP, Galhardo M, Garcia M, Birgel EH. 1992. Avaliação do eritrograma de búfalos (Bubalus bubalis) criados na região do Vale do Ribeira em São Paulo. Braz J Vet Res Anim Sci 29: 113-119.

29. Silva MB, D'Angelino JL, Araujo WP, Galhardo M, Garcia M, Birgel EH. 1992. Leucograma de búfalas criadas no Vale do Ribeira, Sao Paulo. Influencia de fatores raciais e etarios. Braz. J Vet Res Anim Sci 29: 121-129.

30. Verma DN, Lal SN, Singh SP, Parkash OM, Parkash O. 2000. Effect of season on biological responses and productivity of buffalo. J Anim Sci 15: 237-244. 